\title{
Vortices inside a single-stage axial air turbine captured by Particle Image Velocimetry
}

\author{
Daniel Duda ${ }^{1, *}$, Vitalii Yanovych ${ }^{1}$, Václav Uruba ${ }^{1,2}$, Martin Němec ${ }^{3}$, Pavel Žitek ${ }^{1}$ \\ ${ }^{1}$ Faculty of Mechanical Engineering, University of West Bohemia in Pilsen, Univerzitní 22, 30614 \\ Pilsen, Czech Republic \\ ${ }^{2}$ Institute of Thermomechanics, Czech Academy of Sciences, Doleškova 5, 180 00, Prague, Czech \\ Republic \\ ${ }^{3}$ Czech Aerospace Research Centre, Ke Kouli 2, 180 00, Prague, Czech Republic
}

\begin{abstract}
Particle Image Velocimetry (PIV) is an experimental method of fluid research resulting into a spatially resolved two-dimensional velocity field. We measured the velocity inside a single-stage axial test turbine at the Czech Aerospace Research Centre. We studied the axial $\times$ tangential plane just behind the rotor wheel at the tip radius under three regimes: underloaded, designed and overloaded ones. We found individual vortices in the instantaneous snapshots and we analyze their properties statistically.
\end{abstract}

\section{Introduction}

Vortex is a coherent structure typical for turbulent flows [1]. Coherent relates to the motion state of macroscopic number of molecules with motion state correlated over a very large distance (in compared with molecular scale). Vortex is an object [2], which can exist only in the fluid, thus it has some aspects similar to the so-called quasiparticles (phonons, magnons, rotons..., excitation waves in a material), but while quasiparticles are fully delocalized in direct space and localized in reciprocal space, vortex is much closer to localization in direct space, although not fully. It is not possible to count the number of molecules which the vortex consists of, because (i) the vortex influences an infinite area in an infinite fluid domain and (ii) there are always more vortices influencing the single molecule, and (iii) the vortex itself contains other vortices [3]. It carries the energy, it has a finite life-time, it moves through the fluid with some non-zero velocity relative to the surrounding fluid transporting energy and momentum. Therefore, it can be declared it really exists, even in the framework of materialistic philosophy.

From the engineering point of view, the turbulent flow consisting of interacting vortices is unwanted in majority of applications (which is pity), because the interactions of vortices transfer energy from the main flow towards the small scale, where dissipation occurs. Thus the mechanical energy is "lost" to be born downstream in the form of heat. This mechanism is called Richardson cascade [4] and the conservation of energy rate flowing through scales leads to the famous Kolmogorov $k^{-5 / 3}$ law [5].

\footnotetext{
*Corresponding author: dudad@kke.zcu.cz
} 
The flow transits to turbulence via two basic instabilities: the Kelvin-Helmholtz instability between two fluid volumes moving parallel by different velocities (i.e. shear layers), and by the Rayleigh-Taylor instability between two fluids driven by differently strong forces [6]. The later one is famous in connection with the thermal convection, where the difference in density of cooled and heated fluid is responsible for formation of the convective cells or thermal plumes; but the same mechanism applies for the rotating systems [7], which is the case of turbomachinery. The first mentioned instability (Kelvin-Helmholtz) leads to rippling of the interface forming regular pattern of laminar vortices, which later start to interact. The magic feature of this instability is, that vortices contain shear layers as well, thus the rippling repeats inside the formed vortices leading to the fractal appearance. Kelvin-Helmholtz instability is typical for jets and wakes turbulizing their shear layers [8]. The flow past a wheel with blades composites of alternating jets and wakes; wakes form past the blades, jets do past the inter-blade channels. In this article we look at the vortices past a such rotor wheel, where the flow is influenced by the preceding stator wheel as well.

\section{Experiment}

The present measurement was performed at the Czech Aerospace Research Centre, which is located in Prague. They have, among others, a single stage axial air test turbine, whose construction allows relatively easy displacement of blades. The airflow is driven by a powerful radial 12-stage ČKD compressor with input power of 1.3 MW [9].

We use the optical non-intrusive method Particle Image Velocimetry (PIV) [10, 11, 12]. We measure the area of size $28 \times 28 \mathrm{~mm}$ just behind the rotor wheel, the area is oriented in axial $\times$ tangential direction, therefore the wake system is well observable, see Fig. 1. We synchronize our PIV system with the turbine rotations, therefore each of the instantaneous snapshots is captured at the same angular phase of the rotor wheel. The consequence is, that the repeating wake pattern appears on the ensemble average as well, see Fig. 1.

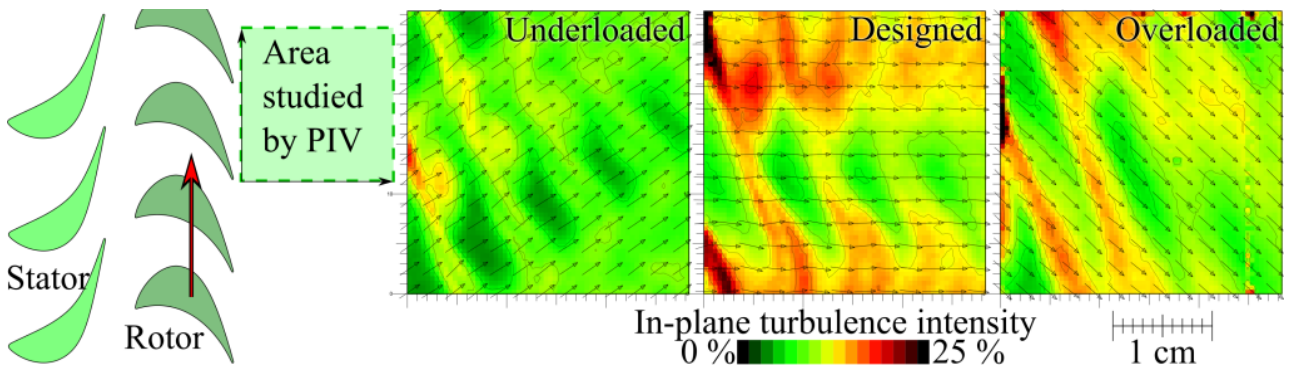

Fig. 1. Sketch of the measured PIV area relative to the stator and rotor wheel. Right part shows the spatial distribution of turbulence intensity calculated from the in-plane velocity components only. Arrows represent the average velocity listed in Table 1. Color version on-line or by using the QR-code.

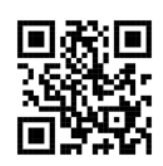

There was prepared two windows allowing the optical access through the steel turbine body, the first one for the camera looking to the area just described, the second one was a bit downstream in the diffusor body and it serves for the laser beam illuminating the particles observed by the camera.

\section{Data processing}

The PIV camera captures a pair of images delayed by few microseconds, during this measurement we used time delay of $5 \mu$ s for the designed regime and $4 \mu$ s for the off-design 
ones. This image pair is processed via the method "Adaptive PIV" inside the "Dantec Dynamic Studio" software resulting into an ensemble of instantaneous velocity fields of two velocity components: the axial one denoted $u$ and the tangential one denoted $v$. The velocity vectors are placed in a regular square grid of $64 \times 64$ cells of size $0.457 \mathrm{~mm}$.

The ensemble of such fields is exported for further processing by a custom-made software. First of all, we cutoff collar of one grid cell, because the quality there is affected by the significant number of particles leaving or entering the field of view during the time delay between consecutive frames.

Second we filter out the datasets with too high energy at smallest length-scale. This algorithm is in more detail described in our article [13]. Of course, it is unwanted to affect a statistical ensemble, but note that this is not a laboratory measurement - it was performed inside a turbine made of shiny steel, the studied fluid was behind multiple Plexiglas windows, the laser sheet was mirrored, the tracing particles condensed on the blade surface creating larger droplets splashing on the window, etc. As a consequence, there is lot of heavily damaged vector fields with large areas of spurious vectors. We do not manipulate the measured vector according to its surroundings, as this would mask some physical small-scale fluctuations, instead we remove the entire snapshot.

In order to highlight the vortices, we remove the spatial average velocity of each snapshot. The ensemble of spatial average velocities is characterized in Table 1. The resulting spatial Reynolds decomposition is displayed in Fig. 2. The comparison with a more standard temporal Reynolds decomposition is discussed in our article [14].

Table 1. Advective velocities, i.e. spatio-temporal averages of axial $(u)$ and tangential $(v)$ velocity components and the standard deviation of the spatial averages. All units are $[\mathrm{m} / \mathrm{s}]$. Subscript "S" signifies spatial average, while " $\mathrm{T}$ " means temporal average or standard deviation

\begin{tabular}{cc|cccc}
\hline Regime & Ratio $u / c$ & $\langle u\rangle_{S, T}$ & $\langle v\rangle_{S, T}$ & $\sigma_{T}\left[\langle u\rangle_{S}\right]$ & $\sigma_{T}\left[\langle v\rangle_{S}\right]$ \\
\hline Underloaded & 0.70 & 28.61 & 20.31 & 0.32 & 0.58 \\
Designed & 0.55 & 29.47 & -1.79 & 0.50 & 0.80 \\
Overloaded & 0.40 & 31.44 & -28.47 & 0.63 & 0.78 \\
\hline
\end{tabular}

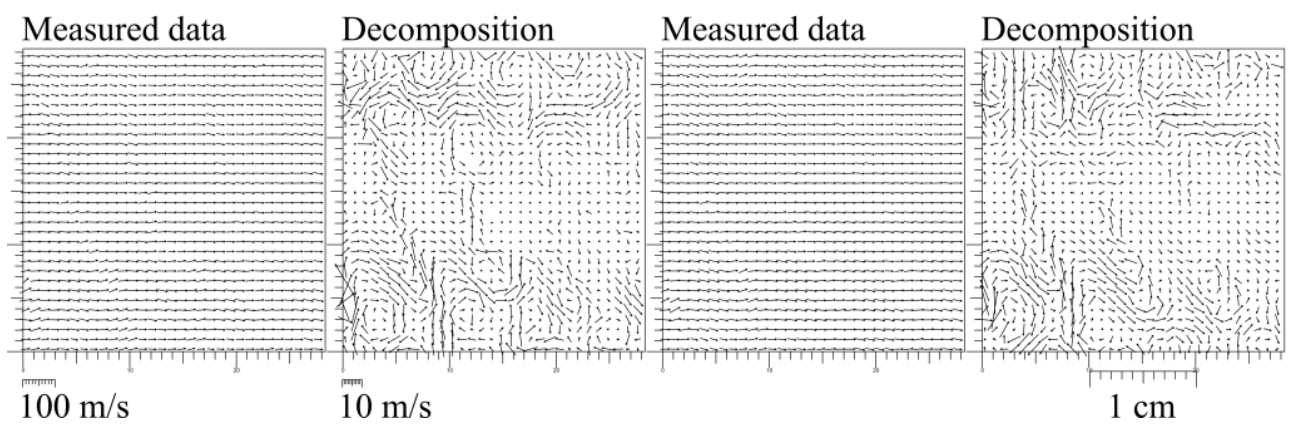

Fig. 2. Example of two instantaneous snapshots with subtracting the advective velocity in order to show the turbulent structures. Both examples are at nominal regime. Only each second arrow is displayed for clear. The scale shows how long would be the arrow of the written velocity (i.e. $100 \mathrm{~m} / \mathrm{s}$ and $10 \mathrm{~m} / \mathrm{s}$ respectively). We see vortices and jets, which are different among snapshots. In the central part, there is a quiet band due to stator jet (note the turbulence intensity in Fig. 1 is lower in this band too). 


\subsection{Searching individual vortices}

The searching of individual vortices is based on the Nelder-Mead "Amoeba" algorithm or "downhill simplex method" [15]. This algorithm finds the minimum energy of the difference of the input velocity field and the velocity field of a vortex in multidimensional space. This space has $N=4$ dimensions characterizing the free parameters of a vortex in two dimensional velocity field: the position of the vortex center ( 2 coordinates), the radius of vortex core and its circulation. In fact, we could introduce more fitting parameters, as the angle of the vortex tube to the studied plane and the orientation of this angle, but we prefer to stay as simple as possible. The algorithm [15] constructs a simplex (simplest body closing finite volume) of $N+1$ points. By trying few styles of step, it moves downhill to the nearest local minimum similarly as the real amoeba would do (here comes the nickname of the algorithm).

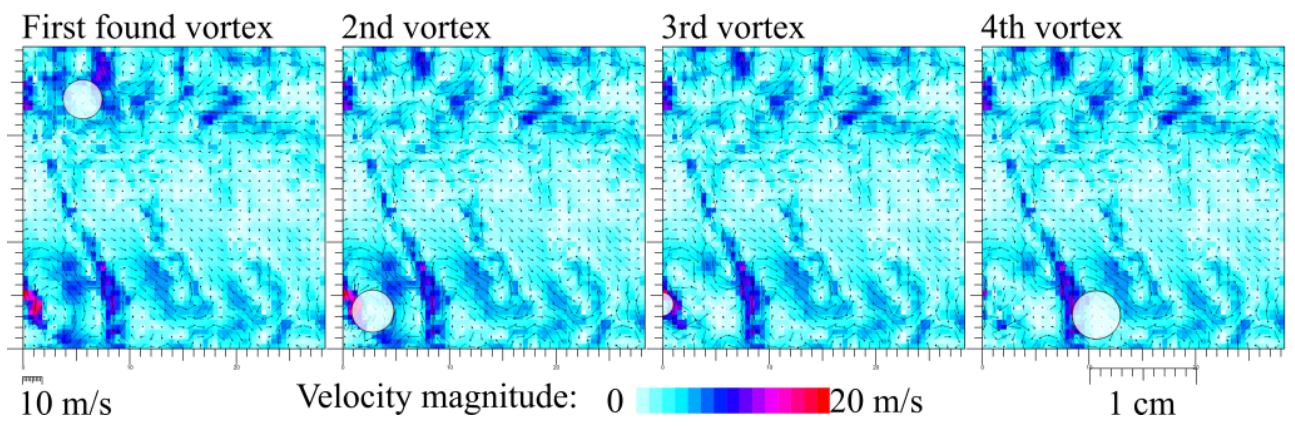

Fig. 3. Example of searching 4 first vortices in a single instantaneous velocity field with subtracted the mean advective velocity. Each vortex partly masks weaker vortices, therefore we subtract it after each successive fit. There is a link to the animated version of this figure up to 50 vortices in the QR-code.

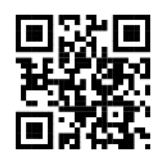

The work flow is as follows: there is found a single vortex in the velocity field, we fall into the local minimum of energy of the remain by using the above mentioned algorithm, then we subtract this vortex from the data and repeat while the energy of new vortex is higher than $1 \cdot 10^{-4}$ times the input energy. First four steps are displayed in Fig. 3 and Fig. 4 shows the input velocity field, the velocity field of vortices and the rest, whose energy is minimized.
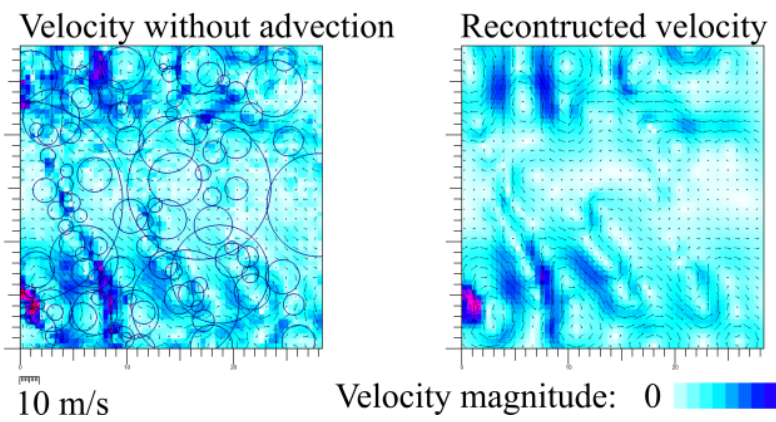

Velocity magnitude: 0

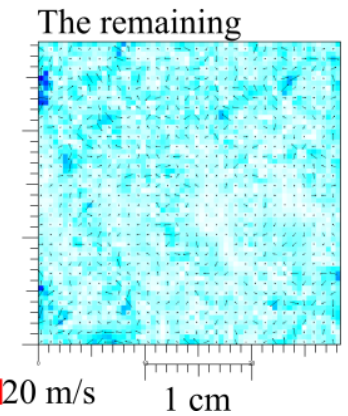

Fig. 4. Example of the instantaneous snapshot (same as in Figs 2 and 3) with the found vortices displayed as circles with radii of the vortex core. Second panel sows the integral velocity field of all these vortices and the third panel represents the rest (difference of first and second panel), i.e. vectors, which were not described by using individual vortices. Color version on-line or by using the QR-code.

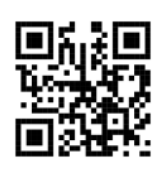

Table 2 and Fig. 5 summarize the convergence of the vortex field to the original data. We can see, that the ensemble of around 130 vortices can explain $77 \%, 74 \%$ and $68 \%$ of the spatial fluctuations. 


\section{Results}

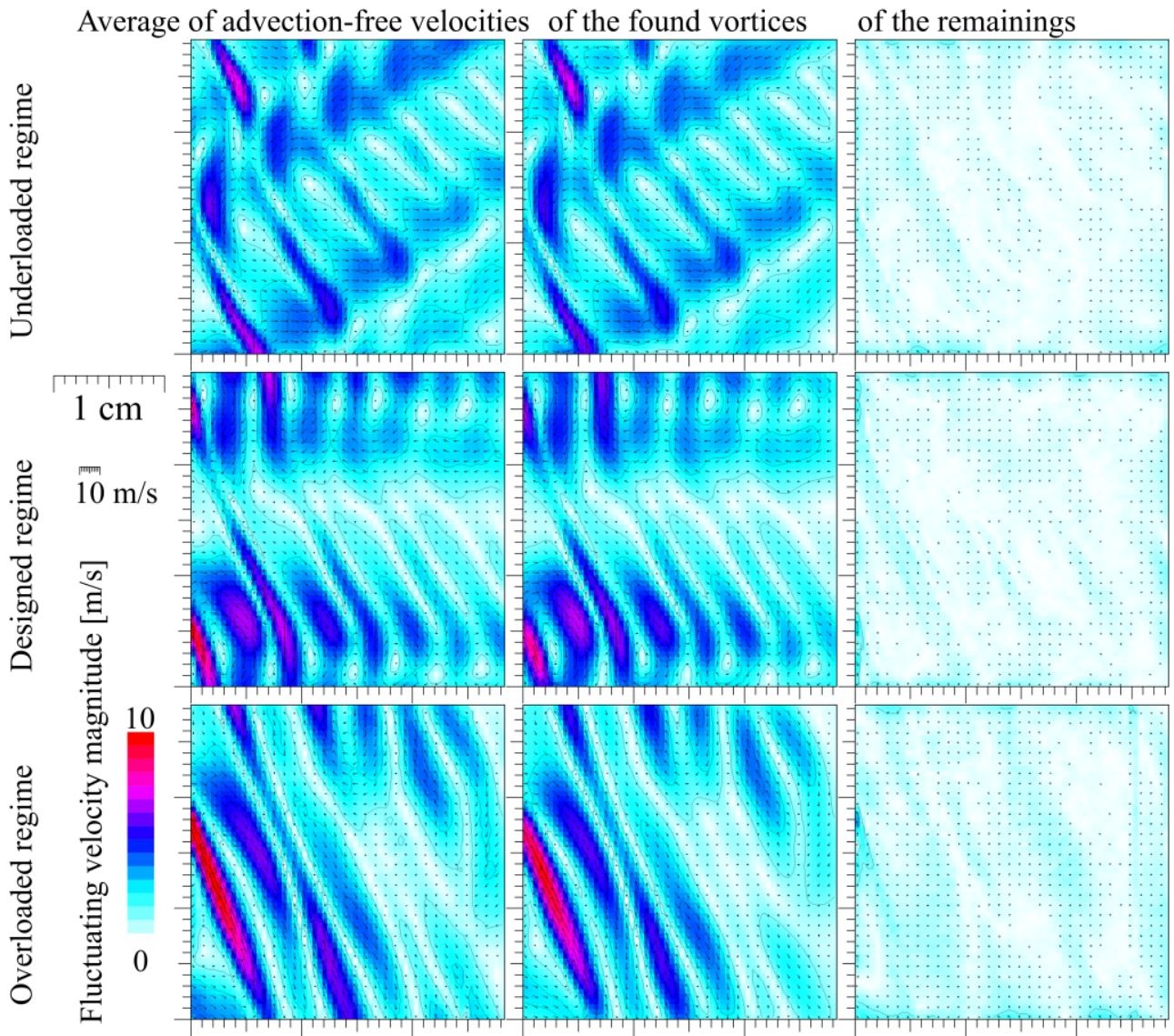

Fig. 5. Table of figures illustrates the ensemble-average of instantaneous velocities without advection (left column), the average velocity field generated by all vortices found there (middle column) and the average of all remaining velocity fields (last column). Rows represent the underloaded (top), designed (middle) and overloaded (bottom) turbine regime. Color version on-line or by using the QR-code.

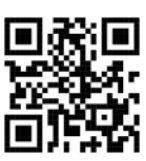

Table 2. Overview of energies. $E_{t}$ refers to the energy of the original velocity field, $E_{S R D}$ is the energy of Spatial Reynolds Decomposition discussed in Fig. 2 and serving as an input into the vortex searching algorithm, $E_{v}$ is the energy of found vortices and $E_{r}$ is that of the remaining. $N_{v}$ denotes the average number of vortices found in each snapshot.

\begin{tabular}{cc|cccc}
\hline Regime & $E_{t}\left[\mathrm{~m}^{2} \mathrm{~s}^{-2}\right]$ & $N_{v}$ & $E_{S R D}\left[\mathrm{~m}^{2} \mathrm{~s}^{-2}\right]$ & $E_{v}\left[\mathrm{~m}^{2} \mathrm{~s}^{-2}\right]$ & $E_{r}\left[\mathrm{~m}^{2} \mathrm{~s}^{-2}\right]$ \\
\hline Underloaded & $623.8 \pm 14.6$ & $139.2 \pm 28.6$ & $8.04 \pm 0.67$ & $6.17 \pm 0.67$ & $1.90 \pm 0.29$ \\
Designed & $447.2 \pm 15.4$ & $131.7 \pm 28.0$ & $10.88 \pm 1.12$ & $8.01 \pm 1.04$ & $2.86 \pm 0.66$ \\
& & & & \\
Overloaded & $914.7 \pm 29.1$ & $122.4 \pm 28.5$ & $14.52 \pm 1.84$ & $9.89 \pm 1.40$ & $4.63 \pm 1.14$ \\
\hline
\end{tabular}


One of the advantage of this approach in comparison with the more standard and easier summation of some scalar parameter is, that the individual vortex, once found, is not lost due to the presence of opposite events. E.g. the appearance of clockwise or counter-clockwise vortices in the pattern of alternating jets and wakes is shown in Fig. 6. There are clearly observable peaks of one or second vortices at shear layers, but the population of the opposite vortices is non-zero at its minima
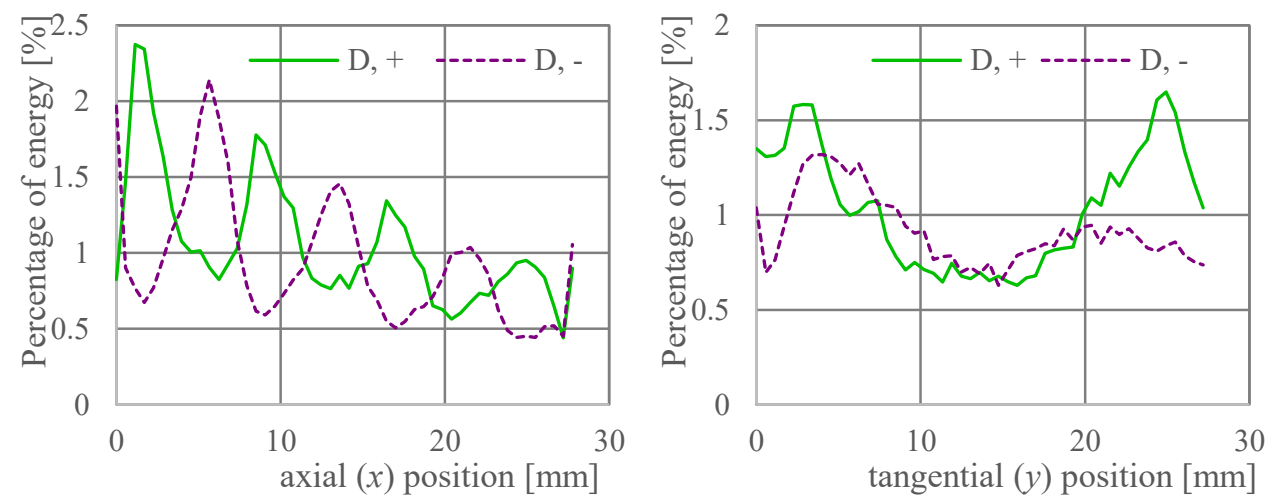

Fig. 6. Distribution of positions of positive (solid green line) and negative (dashed violet) vortices under the designed (D) conditions. The high is normalized via the energy, which the vortex saves from the velocity field.

The distribution of vortex radii is displayed in Fig. 7. We see that all three cases display very similar behavior, except the underload regime contains slightly larger amount of vortices of radius $5 \mathrm{~mm}$. The maximum for all cases lies at about $0.6 \mathrm{~mm}$ and with decreasing radius it scales as $r^{14 / 3}$, while with increasing radius it decreases as $r^{-10 / 3}$. The flatter part in between displays power decay $r^{-1}$. At this moment we lack any theoretical justification for these scalings, additionally we do not know, if these exponents are universal and comparable with e.g. grid turbulence or general wake flows, or if it is a special feature of flow inside an axial turbine. When thinking about the size of vortices, one has to take into account the limit of spatial resolution of the PIV grid, which smooths out any flow structure of such size.

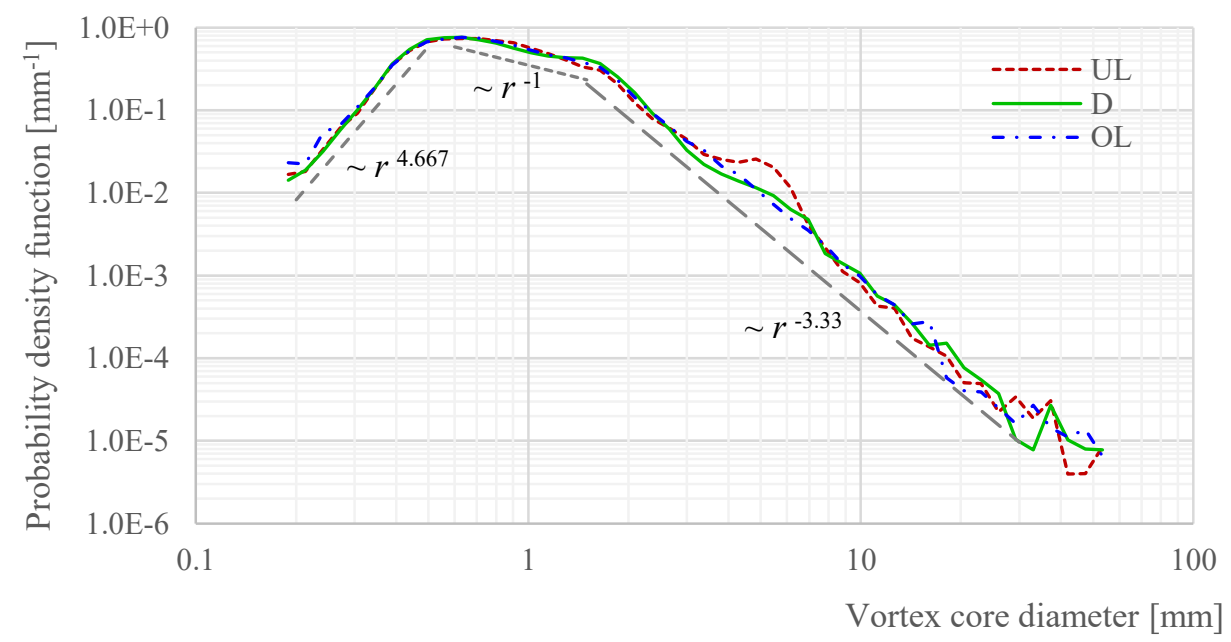

Fig. 7. Probability density function of the vortex core radii. The density is normalized by the energy, which the current vortex saves and by the bin width (hence the unit $\mathrm{mm}^{-1}$ ). UL abbreviates Underloaded regime, $\mathrm{D}$ does the Designed regime and OL plays for Overloaded regime. The grey dashed lines represent the powers $14 / 3,-1$ and $-10 / 3$ respectively. 
The distribution of vortex circulations is displayed in Fig. 8. Distributions of all cases again follow the same shape, but here the maxima are slightly shifted among different cases: the maximum is reached at circulation $\Gamma_{\mathrm{UL}}=0.7 \mathrm{~mm}^{2} / \mathrm{s}$ at the underload regime, $\Gamma_{\mathrm{D}}=0.9 \mathrm{~mm}^{2} / \mathrm{s}$ fir design regime and $\Gamma_{\mathrm{OL}}=1.1 \mathrm{~mm}^{2} / \mathrm{s}$ at the overload regime. This shift might be related to the different average energy of the fluctuations, as the underloaded regime displays smallest fluctuation energy and the overloaded does the highest; see Table 2 for comparison. The data follow power law with exponent +5 / 3 for weaker vortices and it has negative exponent -1 for stronger vortices. For even stronger vortices, this slope increases.

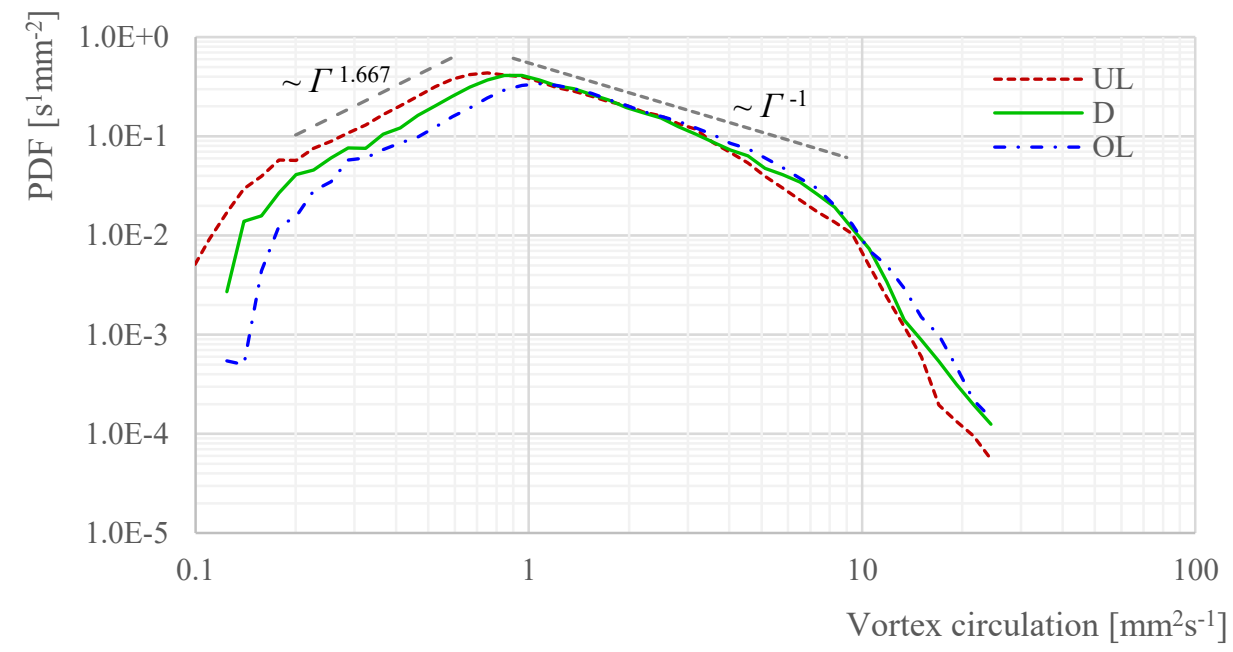

Fig. 8. Probability density function of the vortex circulations; both, negative and positive values are added in the log-log plot. The density is normalized by the energy, which the current vortex saves and by the bin width (hence the unit $\mathrm{s}^{1} \mathrm{~m}^{-2}$ ). The grey dashed lines represent the powers $5 / 3$ and -1 respectively.

This method of searching individual vortices is just new and at this moment, we cannot be sure, that the observed dependencies are real physical effects and not only some property of this algorithm. E.g. the size of vortex core can easily be significantly larger than the field of view, which is mathematically correct, when subtracting such a vortex reduces the energy of the velocity field, but physically it does not make much sense (although such vortices can exist).

Another issue is that the used vortex model expects that each vortex tube crosses the measuring plane perpendicularly, which is not true. The effect of deformed section of the vortex due to non-perpendicular crossing has to be included into the algorithm in future by introducing two more fitting parameters. There is one other issue, which needs to be solved for future use: the ability of reaching global, not only local, energy minima. We plan to perform this by using the method of simulated annealing with some non-zero prescribed temperature.

\section{Conclusion}

By using the method Particle Image Velocimetry (PIV) we measured the ensembles of twodimensional velocity fields inside the axial turbine. We studied the axial $\times$ tangential plane just behind the rotor wheel at the tip radius. We developed a unique algorithm for detecting individual vortices in such a velocity field. We show, that the distribution of vortex radii a circulations are similar across regimes and we observed a new scaling laws, which we are not able to explain yet. 
The in-situ measurement was performed within the framework of the project TN01000007-NCE. The analysis of individual vortices performed by D. D. was supported by the MŠ ZČU program Postdoc 2020.

\section{References}

1. L. Skrbek and K. R. Sreenivasan, Developed quantum turbulence and its decay, Phys. Fluids 24, 011301 (2012)

2. G. Haler, An objective definition of a vortex, J. Fluid Mech. 525, 1-26 (2005)

3. E. Amromin, Analysis of vortex core in steady turbulent flow, Phys. Fluids 19, 118108 (2007)

4. L. F. Richardson, Weather prediction by numerical process (Cambridge University Press, 1922)

5. A.N. Kolmogorov, The local structure of turbulence in incompressible viscous fluid for very large Reynolds numbers, DAN SSSR 30, 299-303 (1941)

6. Y. Zhou, Rayleigh-Taylor and Richtmyer-Meshkov instability induced flow, turbulence, and mixing. I, Phys. Rep. 720-722, 1-135 (2017)

7. M. A. Fardin, C. Perge and N. Taberlet, "The hydrogen atom of fluid dynamics" introduction to the Taylor-Couette flow for soft matter scientists, Soft Matter 10, 35233535 (2014)

8. E. J. List, Turbulent jets and plumes, Ann. Rev. Fluid Mech. 14, 189-212 (1982)

9. T. Jelínek, M Němec, Investigation of unsteady flow in axial turbine stage, EPJ Web Conf. 25, 01033 (2012)

10. C. Tropea, A. Yarin, and J.F. Foss, Springer Handbook of Experimental Fluid Mechanics, (Springer, Heidelberg, DE, 2007)

11. P. Švančara, P. Hrubcová, M. Rotter, and M. La Mantia, Visualization study of thermal counterflow of superfluid helium in the proximity of the heat source by using solid deuterium hydride particles. Phys. Rev. Fluids, 3, 114701 (2018)

12. D. Duda, The PIV study of air flow past the counter-swirler 53983. MATEC Web of Conferences, 16805004 (2018)

13. D. Duda and V. Uruba, Spatial Spectrum from Particle Image Velocimetry Data, ASME J of Nuclear Rad. Sci. 5, 030912 (2019)

14. D. Duda and V. Uruba, PIV of air flow over a step and discussion of fluctuation decompositions, AIP conference proceedings 2000, 020005 (2018)

15. J. A. Nelder and R. Mead, A simplex method for function minimization, The Computer Journal 7, 308-313 (1965) 